\title{
Desarrollo de lenguaje en niños con implante coclear en centro terciario de salud: Serie clínica
}

\author{
Language development in children with cochlear implant \\ at a tertiary health care center: Clinical series
}

\author{
Constanza Díaz S1, Gloria Ribalta L², Marcos Goycoolea V², Felipe Cardemil M², Pilar Alarcón F, \\ Raquel Levy G ${ }^{3}$, Mónica Sierra G ${ }^{3}$, Mauricio Cohen $V^{2}$, Tomás Labatut P², Ernesto Reid G².
}

\begin{abstract}
RESUMEN
Introducción: Desde la década de los 90, los implantes cocleares han progresado desde aplicaciones experimentales a un uso clínico estandarizado en niños con pérdida auditiva severa y profunda. Las investigaciones han producido cambios en la tecnología de los implantes y ha dado lugar a la expansión de los candidatos a implante para incluir a los niños menores de 2 años de edad. El objetivo principal de la implantación coclear pediátrica es facilitar el desarrollo del lenguaje hablado. A pesar de los avances en la tecnología de implantes y los cambios en el manejo de la pérdida auditiva, los resultados de lenguaje en niños son todavía muy variables, participando de este proceso tanto el tratamiento oportuno, la red familiar y nivel socioeconómico entre algunos de ellos.

objetivo: Describir resultados de niños menores 6 años sometidos a cirugía de implante coclear en un centro terciario de salud.

Material y método: Estudio longitudinal de cohorte retrospectivo, análisis a 99 pacientes implantados en centro terciario de salud desde inicio programa en 1994 a 2015.

Resultados: 99 pacientes, 57\% hombres 43\% mujeres, edad promedio implantación 16,3 meses. 54,8\% casos origen congénito no especificado o prematurez. En $74,4 \%$ se objetivó presencia de lenguaje auditivo verbal. El nivel socioeconómico (NSE) mostró asociación con no desarrollo de lenguaje $(p=0,009)$ evidenciando que pacientes de bajo NSE $20 \%$ no desarrolla lenguaje. La variable discapacidad asociada $(p<0,001)$ $y$ la variable $\operatorname{NSE}(p=0,036)$ se asociaron de manera independiente a lenguaje de señas o no desarrollo de lenguaje oral.
\end{abstract}

Conclusión: Los implantes cocleares ofrecen una opción en la rehabilitación auditiva en hipoacusia sensorio neural profunda, para candidatos de todo el espectro de edad, sin embargo los resultados dependen en gran medida del entorno en el que se utilizan los implantes cocleares.

Palabras clave: Implante coclear, desarrollo de lenguaje, infantil, rol familiar, lenguaje oral.

\footnotetext{
Médico General CESFAM Vitacura, Santiago, Chile.

Médico Servicio de Otorrinolaringología Clínica Las Condes, Santiago, Chile.

Tecnólogo Médico de Servicio de Otorrinolaringología Clínica Las Condes, Santiago, Chile.

Recibido el 8 de junio, 2017. Aceptado el 15 de junio, 2018.
} 


\section{ABSTRACT}

Introduction: Since the 90s, cochlear implantation has progressed from experimental to standard clinical practice for children with severe and profound hearing loss. Research has produced changes in implant technology and has led to the expansion of implant candidacy to include children younger than 2 years of age. The primary goal of pediatric cochlear implantation is to facilitate spoken language development. Despite the advances in implant technology and the changes to the management of hearing loss, language outcomes for children are still highly variable, participating in this process both timely treatment, the family network and socio economic level among others.

Aim: describe outcomes of patients younger than 6 years undergoing cochlear implant surgery in a tertiary care center.

Material and method: retrospective longitudinal cohort study, 99 patients implanted in a tertiary health center since the program's inception in 1994 to 2015 was analyzed.

Results: 99 patients, $57 \%$ men $43 \%$ women, average age of implantation 16.3 months. $54.8 \%$ of cases were congenital or prematurity. In $74.4 \%$ they had auditory verbal language. Socioeconomic status (SES) showed association with the lack of language development $(p=0.009)$ demonstrating that low SES patients $(20 \%)$ do not develop language. The disability associated variable $(p<0.001)$ and the SES variable $(p$ $=0.036$ ) were independently associated with sign language or no oral language development.

Conclusions: Cochlear implants offer an option for hearing rehabilitation in profound hearing loss, for candidates of all ages, however the results depend largely on the environment in which cochlear implants are used.

Key words: Cochlear implants, children, language outcomes, family involvement, early diagnosis, auditory-oral.

\section{INTRODUCCIÓN}

La disminución de la capacidad auditiva impacta profundamente la calidad de vida de quien la padece, repercutiendo no solo en la capacidad de comunicación con los pares, sino que también altera el desarrollo social, educacional y finalmente la capacidad de inserción en la sociedad. Es por este motivo que la detección temprana del déficit auditivo, acompañada de la implementación auditiva precoz, es hoy por hoy, la estrategia más eficaz para reducir su impacto. En Chile, la hipoacusia congénita es una patología con una importante incidencia en la población neonatal, siendo incluso más frecuente que el hipotiroidismo congénito y la fenilcetonuria clásica, ambas patologías con tamizaje universal del recién nacido cubiertas por ley AUGE (Acceso Universal de Garantías Explícitas) ${ }^{1}$.

Cerca de 1-2 de cada 1.000 niños nacen con sordera profunda ${ }^{2,3}$. La pérdida de audición, es- pecialmente en sus formas más graves, se asocia con riesgos específicos de desarrollo. Múltiples estudios han demostrado el impacto que tiene el diagnóstico y manejo precoz de esta patología en el desarrollo posterior tanto del lenguaje como del habla, así como en el rendimiento académico y bienestar social. Hoy en día los métodos de tamizaje disponibles permiten establecer un diagnóstico definitivo de la severidad y la naturaleza de la hipoacusia antes de los 3 meses de vida. Tanto la identificación y las intervenciones médicas tempranas han sido perseguidas agresivamente como prioridades de salud pública ${ }^{4}$. Durante la década de 1990, el implante coclear (IC) se hizo disponible como un tratamiento médico para niños con pérdida auditiva sensorio neural severa a profunda. Un IC es un dispositivo que consta de un componente externo que procesa el sonido en señales eléctricas que se envían a un conjunto de receptores y un electrodo interno que estimula el nervio auditiv $0^{4}$. Los IC son ampliamente utilizados en los niños con 
hipoacusia sensorio neural (HSN) y han mostrado ser una gran promesa en la facilitación del lenguaje oral, percepción del habla y reconocimiento; así también como en la atención y el desarrollo del comportamient $0^{5}$. Pese a los notables avances en la tecnología de IC, muchos niños se quedan muy por detrás de sus pares en las habilidades del habla y del lenguaje. Existen enormes diferencias individuales en el grado en que los niños sordos se benefician plenamente de la intervención temprana con implantación coclear. Niños con perfiles audiológicos similares que reciben la misma intervención a menudo tienen diferencias drásticas de comunicación, lenguaje, habilidades sociales, cognitivas, educativas, alfabetización y resultados profesionales ${ }^{6}$.

\section{OBJETIVO}

Analizar las características asociadas al desarrollo del lenguaje en los niños con hipoacusia congénita, implantados antes de los 6 años de vida en Clínica Las Condes, Santiago, Chile (centro terciario de atención de salud). Se intentó identificar factores de riesgo asociados a la hipoacusia congénita que sirvan de predictores de una mala capacidad de reconocer lenguaje en formato abierto, para lograr desarrollo de lenguaje hablado.

\section{MATERIAL Y MÉTODO}

Estudio de serie clínica retrospectiva. Se incluyeron pacientes con hipoacusia prelingual entre 0-6 años sometidos a implante coclear en el periodo 19942015, con seguimiento mínimo 6 meses. El estudio fue aprobado por el Comité de Ética del centro terciario de salud donde fue realizado el estudio.

Las variables fueron recopiladas en una base de datos MS Excel. Se utilizaron descriptores estadísticos generales como promedio, desviación estándar, frecuencias absolutas y relativas. Para evaluar diferencias entre variables cuantitativas se utilizó t de student, y para evaluar asociación entre variables cualitativas se utilizó test exacto de Fisher. Para todos los análisis se consideró significativa un alfa de $5 \%$.

\section{RESULTADOS}

Se evaluaron 99 casos acogidos a los criterios de inclusión del presente estudio. De éstos $57 \%$ fueron hombres y $43 \%$ fueron mujeres. La edad promedio de implantación fue 16,3 meses con una desviación estándar de 13,6 meses. Existió una diferencia significativa en la edad de implantación entre mujeres y hombres, siendo un promedio 18,9 meses y 14,4 meses, respectivamente $(p=0,02)$. En relación a la etiología de la hipoacusia (Figura 1) el $54,8 \%$ de los casos correspondió a hipoacusia congénita no especificada o prematurez, $14,9 \%$ correspondió a TORCH, 12,8\% posmeningitis y $9 \%$ hereditaria sindrómica.

Del total, se obtuvo información respecto a desarrollo de lenguaje en 98 pacientes. En 67 $(74,4 \%)$ se objetivó la presencia de lenguaje auditivo verbal, en 10 casos $(11,1 \%)$ lenguaje total, 6 casos $(6,7 \%)$ lenguaje de señas y en $7(7,8 \%)$ sin desarrollo de lenguaje (Figura 2); de este último

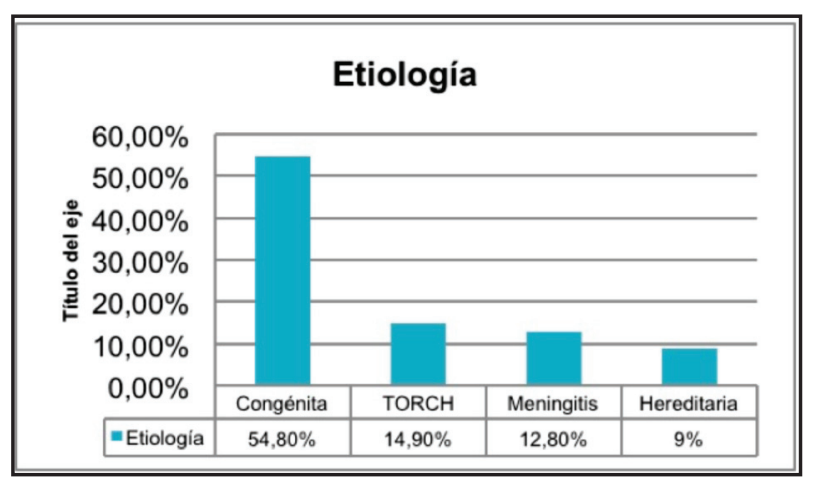

Figura 1. Distribución según etiología. 


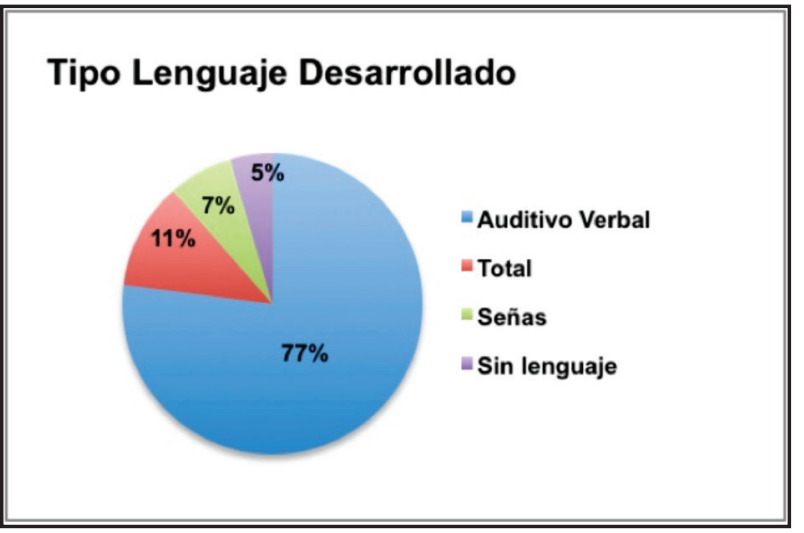

Figura 2. Distribución paciente según lenguaje alcanzado.

grupo cabe destacar que 4 pacientes se encontraban sin seguimiento y 3 casos no eran evaluables aún por su corta edad.

En relación al tipo de lenguaje alcanzado posterior al implante coclear se encontró que un PTP (promedio tonal de tonos puros) posimplante de 22,1 dB tendría asociación con desarrollo de lenguaje auditivo verbal, mientras que un PTP posimplante de 31,6 dB se asociaría con desarrollo de lenguaje de señas $(p=0,01)$. No se encontró asociación entre la discriminación bisílaba $(p=0,718)$ ni en discriminación en oraciones $(p=0,568)$ con el tipo de lenguaje desarrollado. Tampoco se encontró asociación estadísticamente significativa entre el uso de IC bilateral $(p=0,06)$, usuarios de audición bimodal $(p=0,315)$ y desarrollo de lenguaje auditivo verbal. Respecto al tipo de colegio que asisten los niños implantados, 51 (53,1\%) lo hace en colegios normooyente, $23(23,9 \%)$ se dirige a colegios con integración, 8 casos $(8,3 \%)$ va a escuela de sordos, 8 casos $(8,3 \%)$ no acude de los cuales 2 no lo hacen por edad muy pequeña,
3 niños (3,1\%) acude a colegio de educación diferencial o discapacitados y solo 3 casos $(3,1 \%)$ no tenían registro de seguimiento (Tabla 1).

En relación a los factores asociados al tipo de colegio que asiste cada niño se encontró asociación estadísticamente significativa con PTP post IC ( $p \leq 0,0003$ ), encontrando que aquellos pacientes que asisten a escuelas normooyentes poseen un PTP de 22,6 dB versus aquellos que asisten a escuelas de sordos con un PTP de 32,2 $d B(p=0,003)$. Respecto al nivel educacional alcanzado (Figura 3), 40 casos $(43,01 \%)$ poseen escolaridad básica, 24 casos $(25,8 \%)$ enseñanza media, 7 casos $(7,5 \%)$ educación universitaria, 5 casos $(5,4 \%)$ educación técnica superior, 12 casos (12,9\%) acuden a preescolar, 2 casos $(2,15 \%)$ sin seguimiento y en 3 casos $(3,2 \%)$ no es evaluable.

Es importante mencionar que muchos de los pacientes catalogados en el grupo de preescolares, escolaridad básica y media caen en estas categorías pues actualmente, se encuentran cursando este grado académico, lo cual no sig-

Tabla 1. Distribución implantados según colegio al que asisten

\begin{tabular}{|l|cc|}
\hline Tipo colegio & $N^{0}$ niños & $\%$ \\
\hline Normooyente & 51 & 53,1 \\
Con integración & 23 & 23,9 \\
Escuela sordos & 8 & 8,3 \\
No acude & 8 & 8,3 \\
Diferencial & 3 & 3,1 \\
Sin seguimiento & 3 & 3,1 \\
\hline
\end{tabular}




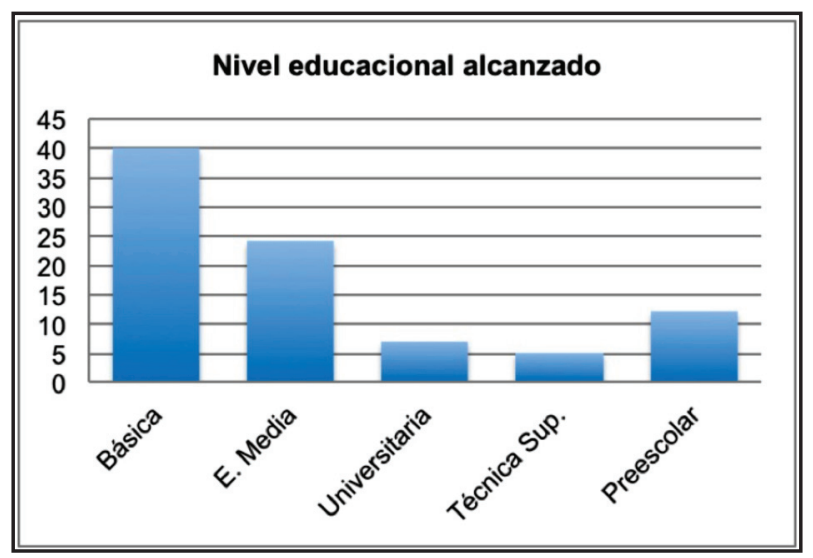

Figura 3. Distribución según nivel educacional alcanzado.

nifica que sea el grado máximo de escolaridad que hayan alcanzado. Al analizar el nivel educacional alcanzado, se observó que este presenta asociaciones estadísticamente significativas con bimodalidad $(p=0,005)$ y discriminación bisílaba $(p=0,007)$.

En relación a la implementación bimodal podemos ver que de aquellos que cursan nivel preescolar $41,7 \%$ está implementado bimodalmente, en enseñanza básica $30 \%$, en enseñanza media $20,8 \%$ y en nivel técnico y universitario $0 \%$ de los pacientes. Esta diferencia en el uso de la bi-



Figura 4. Distribución según nivel socioeconómico de los pacientes implantados infantiles. modalidad entre los escolares más jóvenes y los universitarios se debe a que éstos últimos no se les insistió en la bimodalidad pues en la época en que fueron implantados, hace ya muchos años, no se consideraba esta práctica tan importante como se le considera hoy.

Se analizó la distribución y comportamiento según nivel socioeconómico (NSE) (Figura 4), observando que 51 pacientes $(53,1 \%)$ pertenecieron a un estrato socioeconómico alto, $40(41,6 \%)$ a un estrato medio y 5 pacientes $(5,21 \%)$ a un nivel bajo.

Se encontró que el nivel socioeconómico tendría asociación estadísticamente significativa con asistir a escuela de sordos $(p=0,04)$ mostrando que $20 \%$ de los niños pertenecientes al grupo de NSE bajo acude a escuela de sordos, aquellos con NSE medio $15 \%$ y aquellos con NSE alto solo 1,9\%. A su vez el NSE mostró asociación con el no desarrollo de lenguaje ( $p$ $=0,009$ ) evidenciando que en aquellos pacientes de NSE bajo el $20 \%$ no desarrolló lenguaje, NSE medio $8,1 \%$ no desarrolló lenguaje mientras que en NSE alto solo 6,3\% de los pacientes no desarrolló lenguaje.

Al realizar una regresión logística (Tabla 2) para evaluar el riesgo de presentar lenguaje de señas 0 no desarrollar lenguaje en niños diagnosticados antes de los 6 años, se encontró que la variable discapacidad asociada (Odds ratio 0,008 - $p<0.001$ ) y la variable nivel socioeconómico (Odds ratio 0,16 $-p=0,036$ ) se asociaron de manera independiente a lenguaje de señas o no desarrollo de lenguaje. 
Tabla 2. Regresión logística para evaluar riesgo de presentar lenguaje de señas o no desarrollar lenguaje

\begin{tabular}{|lccc|}
\hline Variable & Odds ratio & Valor $p$ & IC $95 \%$ \\
\hline Sexo & 0,97 & 0,981 & $0,08-10,6$ \\
Diagnóstico & 1,73 & 0,052 & $0,99-3,02$ \\
N ${ }^{0}$ Electrodos & 12,7 & 0,095 & $0,64-253,6$ \\
Malformación coclear & 0,55 & 0,63 & $0,04-6,26$ \\
Discapacidad asociada & 0,008 & $<0,001$ & $0,0005-0,121$ \\
Nivel socioeconómico & 0,161 & 0,036 & $0,02-0,88$ \\
\hline
\end{tabular}

\section{DISCUSIÓN}

La hipoacusia es la discapacidad congénita más común, tres veces más frecuente que el síndrome de Down ${ }^{7}$, con una incidencia general estimada que oscila entre uno y dos casos entre 1.000 nacidos vivos; la infección congénita por citomegalovirus y mutaciones de conexinas son las dos principales causas de la sordera al nacer, mientras que las causas de acueducto vestibular dilatado con la infección congénita por citomegalovirus son las principales causas de la pérdida auditiva prelocutiva que no se expresa en el nacimiento ${ }^{8}$. El diagnóstico precoz e intervención temprana permiten un desarrollo social y educacional normal en el niño, previniendo severas repercusiones lingüísticas y psicosociales. Intervenciones antes de los 6 meses permiten que niños hipoacúsicos tengan un normal desarrollo del habla y del lenguaje, a la par de sus compañeros normooyentes ${ }^{9}$.

La estrategia de realizar tamizaje auditivo universal es la única alternativa realmente efectiva en la pesquisa de la hipoacusia congénita y su aplicación se ha ido generalizando a nivel mundial a partir de la década de 1990. Antes de la introducción del tamizaje auditivo universal, la edad promedio de diagnóstico de una hipoacusia congénita era de 2 años y medio. En el caso de hipoacusias leves, éstas podían incluso pasar desapercibidas hasta la edad escolar ${ }^{6}$. Tanto diversos estudios como la práctica clínica han demostrado que a edades más tempranas de la implantación (<2 años) se producen mayores niveles de rendimiento, mientras que edades posteriores de implantación se asocian con mayores probabilidades de retrasos en el lenguaje, en particular en los subdominios de la gramática y la pragmática ${ }^{10}$. Existen varios factores que pueden predecir la capacidad de reconocer lenguaje en formato abierto que es indispensable para el desarrollo de lenguaje hablado tales como: Edad temprana de implante coclear <2 años, comunicación auditivo verbal, procesador al día. Por otro lado, predicen dificultad en desarrollo lenguaje formato abierto: déficit cognitivo, retraso desarrollo psicomotor y cócleas anormales y/o hipoplasia nervio coclear entre los factores ya conocidos.

Intentos de comprender mejor las fuentes de variabilidad en el habla y el desarrollo del lenguaje después de la implantación coclear, han conducido al descubrimiento de otras áreas del funcionamiento neurocognitivo que se verían afectadas de manera adversa. Kronenberger y cols ${ }^{11}$ reportaron retrasos en tres grandes dominios de la función ejecutiva (FE) en niños con implante coclear: 1) La memoria de trabajo, 2) La velocidad de fluidez (velocidad de procesamiento durante las operaciones cognitivas que requieren atención controlada con esfuerzo) y 3) La concentración-inhibición (la capacidad de mantener un nivel constante de atención para controlar las respuestas). Un período de sordera profunda durante los primeros períodos críticos del desarrollo cerebral, combinado con una pobre experiencia auditiva, puede afectar a amplias áreas de dominio general del desarrollo neurocognitivo $^{12}$. Como resultado, la privación de experiencias auditivas tempranas y actividades puede influir en el desarrollo de las funciones cognitivas y neurobiológicas más básicas y elementales, extendiéndose mucho más allá de la lengua oral.

Un gran número de investigaciones han demostrado que las habilidades de $\mathrm{FE}$, como la memoria de trabajo y el control cognitivo son críticos para el desarrollo del habla y el lenguaje en niños. Idioma y FE son dependientes entre sí 
para el desarrollo, particularmente en el periodo de la infancia ${ }^{13}$. De las tres grandes áreas de la FE identificadas como en riesgo en usuarios de IC a largo plazo, la memoria de trabajo ha recibido la mayor atención pues muestra asociaciones con el lenguaje en niños con IC. La capacidad de memoria de trabajo se asocia significativamente con la capacidad de percepción del habla, la gramática, vocabulario, la lectura y la comunicación conversacional ${ }^{3,14}$. Los individuos con más rápido y más eficiente trabajo de habilidades de memoria verbal son capaces de codificar, almacenar, procesar y recuperar más fonemas y representaciones léxicas de señales de voz, lo que lleva a un mejor desarrollo del lenguaje; del mismo modo las habilidades lingüísticas más fuertes hacen que la codificación verbal y la memoria de trabajo sean más rápidas y más eficientes, siendo la relación entre el lenguaje y la memoria de trabajo verbal más fuerte para los usuarios de IC que para sus pares normooyentes ${ }^{14}$.

Por otro lado, entre las grandes diferencias existentes en niños implantados, un factor importante que moldea el desarrollo de ellos es el entorno familiar en el que se desenvuelven. La mayoría de las investigaciones sobre el tema han hecho hincapié en el papel de la familia en el tratamiento, apoyo en el hogar, tamaño de la familia, nivel de educación, estatus socioeconómico y varios factores maternos incluyendo el apego y la sensibilidad ${ }^{15-18}$. El grado de estimulación a los 12 meses determinaría en forma importante el desarrollo lingüístico, marcando diferencias importantes en edades de implantación tan pequeñas, esto confirma la gran importancia del entorno y en particular de la familia en la rehabilitación del niño implantado con gran influencia en la función ejecutiva y las habilidades del lenguaje hablado ${ }^{19-21}$; grados más altos de la estructura y organización dentro de la familia parece promover un mejor vocabulario, mientras que las familias que funcionan a través del control estricto y reglas rígidamente establecidas pueden de hecho tener el efecto contrario en el desarrollo del vocabulario. Niños en edad preescolar tienen menos problemas con el control emocional cuando las familias reportaron niveles más altos de apoyo y niveles más bajos de conflicto $0^{4,21}$.

Tanto la sensibilidad materna y la estimulación de los padres pueden ser responsables de la variabilidad en estos resultados. Modelos de aprendizaje de lenguaje temprano han subrayado desde hace tiempo el papel clave desempeñado por las interacciones con los primeros cuidadores en la facilitación de la intención comunicativa y los marcos de atención, preparando el escenario para el aprendizaje imitativo de expresión. Las consecuencias de una interacción pobre incluyen apego menos seguro, dificultad para mantener la atención, y un desarrollo más lento de las competencias comunicativas. El comportamiento de los padres, incluyendo la educación materna, estimulación cognitiva y estimulación lingüística, afectarían significativamente el desarrollo del lenguaje oral después de considerar la experiencia auditiva temprana del niño. A los 48 meses después de la implantación, los hijos de padres con mayor educación materna sólo muestran un retraso en el lenguaje de 1,3 años, en comparación con el retraso de 2,7 años en los hijos de padres con educación materna baja. Los hijos de padres tanto con alta sensibilidad materna y alta estimulación lingüística, sólo muestran un retraso de 1 año en el lenguaje, en comparación con 2,5 años en otros grupos donde la sensibilidad materna es baja pese a una alta estimulación cognitiva 0 lingüística ${ }^{23}$.

Del mismo modo, es importante tener presente que los niños con HSN, presentan tasas más altas de problemas de comportamiento exteriorizado, tales como la falta de atención y la agresión (30\%-38\%) que los niños con audición normal $(3 \%-18 \%)^{4}$. Los padres de niños sordos también reportan más problemas de internalización (por ejemplo: ansiedad, tristeza) en comparación con los padres de niños sin dificultades auditivas (25\%-38\% vs $2 \%-17 \%)^{4}$. Usualmente la estimulación recibida suele ser muy alta en el comienzo debido a que los padres se encuentran muy motivados y con altas expectativas por el IC, pero con el paso del tiempo no todas las familias logran el mismo nivel de estimulación ${ }^{19}$. La pérdida de la audición de un niño lo afecta a él, así como a su familia. La crianza de niños sordos o con problemas de audición presenta desafíos únicos a largo plazo que puede colocar a los padres en un mayor riesgo de niveles elevados de estrés. El estrés de los padres se puede definir como una reacción psicológica adversa a las demandas de ser padre, este tipo específico de estrés surge cuando las 
percepciones de las exigencias de la paternidad de los padres superan sus recursos, lo que pone en riesgo los resultados del desarrollo del lenguaje ${ }^{24}$. El entorno familiar es dinámico y puede ser modificado con la educación y la terapia; Ios equipos de rehabilitación deberían recomendar que las familias buscaran educación y/o asesoramiento en estrategias específicas con intervenciones tempranas para modificar su dinámica familiar y apoyar sus necesidades y así facilitar los mejores resultados posibles para sus hijos ${ }^{5,24}$. Nótese que la familia puede crear condiciones óptimas de múltiples formas: facilitándole la integración social, creando dinámicas de interacción que faciliten el acceso al input, implicándose en las actividades de rehabilitación, o solucionando los problemas técnicos asociados al IC. Por todo ello la familia es tan importante. Pero también por algo más: nadie más puede realizar esas funciones ${ }^{19}$.

Otro tema conflictivo y en boga que podría determinar la variabilidad de respuesta entre niños implantados es el aprender lenguaje de señas, expertos coinciden en que un niño debe ser expuesto a un lenguaje accesible de forma regular y frecuente antes de los 5 años de edad para desarrollar la competencia lingüística completa ${ }^{25}$. Estudios indican que los niños con IC educados con algún tipo de lenguaje de señas tienen niveles significativamente más altos de vocabulario que aquellos educados exclusivamente por vía oral ${ }^{9}$. Informaron que una transición de la señal para la comunicación hablada era dependiente de la edad de la implantación. Los niños implantados antes de los 18 meses de edad fueron capaces de hacer la transición. Si la implantación se produce después de 30 meses de edad, la probabilidad de transición de la señal a voz es significativamente reducida. Se sugiere la lengua de señas temprana, por corto tiempo como un puente para el lenguaje hablado, pues sería beneficioso ${ }^{25}$. De esta manera la comunicación multimodal de inicio temprano muestra ser el enfoque más natural los primeros años ${ }^{26,27}$. El uso de un lenguaje de signos facilita el aprendizaje de la lengua a través del uso de medios no auditivos. El niño es capaz de asociar lo que él o ella escucha a través del implante con las representaciones formadas del desarrollo, portando un idioma de apoyo a la lengua hablada ${ }^{18}$.
La elección de educación integrativa tras el implante coclear se asocia con una mayor inteligibilidad de la voz y mejores puntuaciones de lectura. Con la implantación coclear precoz y la intervención educativa especial a los 2 años de edad, muchos niños sordos jóvenes están listos para asistir a escuela normooyente, exhibiendo habla y habilidades del lenguaje que se aproximan a los compañeros de audición normal ${ }^{28}$. En los casos de HSN bilateral, se debe estimular los dos oídos para proporcionar la información auditiva, con el fin de proporcionar los beneficios potenciales de la audición biaural ${ }^{9,29}$.

\section{CONCLUSIÓN}

Se puede concluir con cifras claras en base a un programa de implante coclear con más de 20 años desde su implementación, que más del $70 \%$ de los pacientes implantados desarrolla lenguaje auditivo verbal. Sin duda el PTP posterior a la implantación se relacionaría con el tipo de lenguaje alcanzado y con el tipo de colegio al cual asiste el niño. Tras una exitosa implantación, bajo los criterios adecuados sumado a una correcta estimulación y seguimiento, gran porcentaje de los niños presentes en el estudio $(77 \%)$, logra integrarse a colegios normooyente con o sin integración. Se debe hacer énfasis como tratantes en el uso de bimodalidad pues mostró asociación con mayor nivel educacional por ende mejor desarrollo de lenguaje.

Si bien, la discapacidad asociada y nivel socioeconómico se asociaron de manera independiente a no desarrollo de lenguaje, hay que advertir a los padres y educadores sobre la posibilidad de complementar con comunicación total para que se brinden mejores posibilidades de comunicación. La presencia de NSE alto "protege" del no desarrollo de lenguaje, sin embargo, se debe educar y estimular a los padres a participar y cumplir con las terapias de los niños implantados, así como también en la importancia del núcleo y dinámica familiar sobre resultados que pueda tener el paciente.

Los implantes cocleares ofrecen una opción en la rehabilitación auditiva en HNS profunda, para candidatos de todo el espectro de edad, sin embargo, los resultados dependen en gran 
medida del entorno en el que se utilizan los implantes cocleares, produciendo una amplia gama de variabilidad individual en los resultados. Hoy en día los criterios para la implementación de dicho dispositivo se centran en los beneficios funcionales proporcionados por la amplificación.

\section{BIBLIOGRAFÍA}

1. www.auge.cl

2. Molini E, Calzolaro L, Lapenna R, Ricci G. Universal newborn hearing screening in Umbria region, Italy. Int J Pediatr Otorhinolaryngol 2016; 82: 92-7.

3. Nazar MG, Goycoolea VM, SG, Miguel J, Ried GE, Sierra GM. Evaluación auditiva neonatal universal: Revisión de 10.000 pacientes estudiados. Rev Otorrinolaringol Cir Cabeza Cuello 2009; 69: 93-102.

4. Kronenberger WG, Colson BG, Henning SC, Pisoni DB. Executive Functioning and Speech-Language Skills Following Long-Term Use of Cochlear Implants. J Deaf Stud Deaf Educ 2014; 19: 45670.

5. Cruz I, Vicaria I, Wang NY, Niparko J, Quittner $\mathrm{AL}$; $\mathrm{CD}$ a $\mathrm{Cl}$ Investigative Team. Language and behavioral outcomes in children with developmental disabilities using cochlear implants. Otol Neurotol 2012; 33: 751-60.

6. Holt RF, Beer J, Kronenberger WG, Pisoni DB. Developmental effects of family environment on outcomes in pediatric cochlear implant recipients. Otol Neurotol Off Publ Am Otol Soc Am Neurotol Soc Eur Acad Otol Neurotol 2013; 34: 388-95.

7. Smith RJ, Bale JR JF, White KR. Sensorineural hearing loss in children. The Lancet 2005; 365: 879-90.

8. Nance WE, Lim BG, Dodson KM. Importance of congenital cytomegalovirus infections as a cause for pre-lingual hearing loss. J Clin Viro/2006; 35: 221-5.

9. MD. Rehabilitation of deaf children with a cochlear implant. Rev Med Suisse 2009; 5: 1933-5.

10. Tobey EA, Thal D, Niparko JK, Eisenberg LS, Quittner AL, Wang N-Y, et al. Influence of implantation age on school-age language
No es sólo tecnología, la cirugía, y la instalación; hay mucho trabajo por hacer después de la implantación. 0 como Sue Archbold (La Fundación del oído, Reino Unido) siempre dice: "Lo que los cirujanos empiezan, los educadores tienen que terminar".

performance in pediatric cochlear implant users. Int J Audiol 2013; 52: 219-29.

11. Ruffin CV, Kronenberger WG, Colson BG, Henning SC, PIsoni DB. Long-term speech and language outcomes in prelingually deaf children, adolescents and young adults who received cochlear implants in childhood. Audiol Neurootol 2013; 18: 289-96.

12. Beer J, Kronenberger WG, Pisoni DB. Executive function in everyday life: implications for young cochlear implant users. Cochlear Implants Int 2011; 12: S89-91.

13. Gathercole SE, Baddeley AD. Working Memory and Language. Hove, UK; Hillsdale, USA: Psychology Press; 1993. 280 p.

14. Ylvisaker M, DeBonis D. Executive Function Impairment in Adolescence: TBI and ADHD. Topics in Language Disorders 2000; 20: 29-57.

15. Vanormelingen L, De MaeYer S, Gillis S. Interaction patterns of mothers of children with different degrees of hearing: normally hearing children and congenitally hearing-impaired children with a cochlear implant. Int J Pediatr Otorhinolaryngol 2015; 79: 520-6.

16. Pratt SR. Nonverbal play interaction between hearing mothers and young deaf children. Ear Hear 1991; 12: 328-6.

17. Pressman L, Pipp-Siegel S, Yoshinaga-Itano C, Deas A. Maternal sensitivity predicts language gain in preschool children who are deaf and hard of hearing. J Deaf Stud Deaf Educ 1999; 4: 294304.

18. GEERS AE. Factors influencing spoken language outcomes in children following early cochlear implantation. Adv Otorhinolaryngol 2006; 64: 50-65.

19. Moreno-Torres I, Cid M del M, Santana R, Ramos Á. Estimulación temprana y desarrollo lingüístico en niños sordos con Implante coclear: el primer año de experiencia auditiva. Rev Investig En Logop Res Speech Lang Ther 2011; 1: 56-75. 
20. Yanbay E, Hickson L, Scarinci N, Constantinescu G, Dettman SJ. Language outcomes for children with cochlear implants enrolled in different communication programs. Cochlear Implants Int 2014; 15: 121-35.

21. Langereis M, Vermeulen A. School performance and wellbeing of children with $\mathrm{Cl}$ in different communicative-educational environments. Int J Pediatr Otorhinolaryngol 2015; 79: 834-9.

22. Osto.ıc $S$, DJokovic $S$, Radic-šestic M, Nikolic M, Mikic B, MIRIC D. Factors contributing to communication skills development in cochlear implanted children. Vojnosanit Pregl 2015; 72: 683-8.

23. Quittner AL, Cruz I, Barker DH, Tobey E, Eisenberg LS, Niparko JK, et AL. Effects of maternal sensitivity and cognitive and linguistic stimulation on cochlear implant users' language development over four years. J Pediatr 2013; 162: 343-8.

24. Sarant J, Garrard P. Parenting stress in parents of children with cochlear implants: relationships among parent stress, child language, and unilateral versus bilateral implants. J Deaf Stud Deaf Educ 2014; 19: 85-106.
25. Napoli DJ, Mellon NK, Niparko JK, Rathmann C, Mathur G, Humphries T, et al. Should All Deaf Children Learn Sign Language? Pediatrics 2015; 136: $170-6$.

26. Tian Y, Zhou H, Zhang J, Yang D, Xu Y, Guo Y. [Effect of rehabilitation for prelingual deaf children who use cochlear implants in conjunction with hearing aids in the opposite ears]. Lin Chuang Er Bi Yan Hou Tou Jing Wai Ke Za Zhi 2012; 26 : 868-70, 873.

27. BECKER F. [Only spoken language or sign language?]. Tidsskr Den Nor Lægeforen Tidsskr Prakt Med Ny Række 2012; 132: 136.

28. Connor CM, Hieber S, Arts HA, Zwolan TA. Speech, vocabulary, and the education of children using cochlear implants: oral or total communication? J Speech Lang Hear Res JSLHR2000; 43: 1185204.

29. Kane M0, Schopmeyer B, Mellon NK, Wang NY, Niparko JK. Prelinguistic communication and subsequent language acquisition in children with cochlear implants. Arch Otolaryngol Head Neck Surg 2004; 130: 619-23. 